\title{
Detection of liver metastases from colorectal carcinoma: Is there a place for routine computed tomography arteriography?
}

\author{
Bart van Ooijen, MD, PhD, Matthijs Oudkerk, MD, PhD, Paul I. M. Schmitz, PhD, \\ and Theo Wiggers, MD, PhD, Rotterdam, The Netherlands
}

\begin{abstract}
Background. A prospective evaluation of the liver by preoperative ultrasonography, conventional computed tomography (CT), and continuous CT angiography (CCTA) was performed in 60 patients with primary or secondary colorectal carcinoma.

Methods. The standards of reference were palpation of the liver and intraoperative uitrasonography. The imaging techniques were assessed independently of each other.

Results. In 37 patients 105 liver metastases were identified; 23 patients had no metastases. CCTA had a high sensitivity of $94 \%$ (99 lesions identified) in contrast to ultrasonography (48\%) and conventional CT (52\%). The superiority of CCTA was also manifest in lesions less than $1 \mathrm{~cm}$ in diameter. However, the high sensitivity was accompanied by a high false-positive rate, particularly because of variations in the perfusion of normal liver parenchyma. Overall, CCTA had the highest accuracy (74\%) compared with ultrasonography and CT (both 57\%). The data indicate that preoperative ultrasonography and conventional CT have low sensitivity in the detection of liver metastases.

Conclusioms. Although CCTA seems to be superior to other preoperative imaging techniques, the too low specificity will hamper its routine application in patients with hepatic metastases from colorectal carcinoma. (Surgery 1996;119:511-6.)
\end{abstract}

From the Departments of Surgical Oncology, Radiology, and Statistics, Dr. Daniel den Hoed Cancer Center, Rotterdam, The Netherlands

ONE OF THE MOST IMPORTANT prognostic factors determining survival among patients undergoing hepatic resection for colorectal metastases is the number of metastatic deposits in the liver. ${ }^{1-3}$ There is common agreement to resign from resection when the number of metastases is more than three or four. ${ }^{2,3}$ Therefore precise evaluation of number (and location) is essential to prevent needless exploration. During the past decade the standard modalities for preoperative evaluation of the liver have been computed tomography (CT) and ultrasonography; ${ }^{4,5}$ however, these methods have low efficacy in predicting actual disease state compared with intraoperative ultrasonography (IOUS) ${ }^{6-8}$ At present other preoperative imaging techniques are being investigated, including angiography, CT angiography, and magnetic resonance imaging. ${ }^{9-13}$ In this study we de-

Accepted for publication Sept. 12, 1995.

Reprint requests: B, van Ooijen, MDPhD, Department of Surgical Oncology, Dr. Daniel den Hoed Kliniek, Groene Hilledijk 301, 3075 EA Rotterdam, The Netherlands.

Copyright (C) 1996 by Mosby-Year Book, Inc.

$0039-6060 / 96 / \$ 5.00+0 \quad \mathbf{1 1 / 5 6 / 6 9 4 6 2}$ scribe the application of an angiographic diagnostic approach with a much higher sensitivity for diagnosis of hepatic metastatic disease than conventional CT and ultrasonography. Whether this technique should be added to the standard modalities for preoperative evaluation of hepatic disease is discussed.

\section{PATIENTS AND METHODS}

Sixty consecutive patients with a primary or secondary carcinoma of the colon or rectum (with or without liver involvement) and selected for laparotomy on the basis of conventional CT scans were the subject of the study. Patients were excluded from further investigations when four or more metastases were seen, when extrahepatic disease was present, or when a central localization of metastases made resection technically impossible. Sixty-two eligible patients had given consent during a 30-month period in the Dr. Daniel den Hoed Cancer Center. One patient was withdrawn from the study because peritoneal carcinomatosis made it impossible to perform intraoperative ultrasonography of the liver; one patient was withdrawn because it was impossible to perform an angiography (anxiousness of pa- 


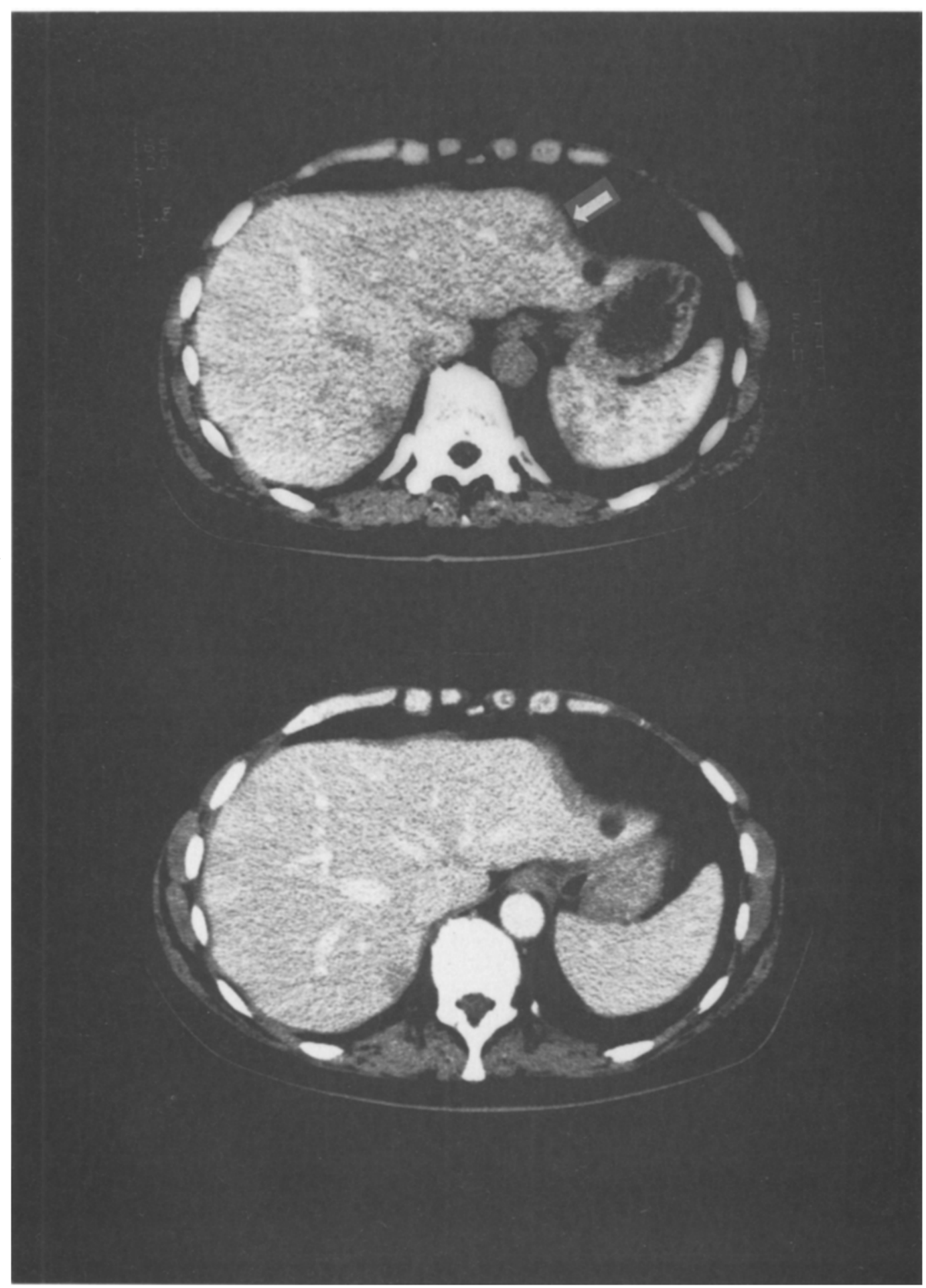

Fig. 1. Conventional CT (lower) and CCTA (upper) scans of same patient. CCTA revealed additional lesion in segment 2 (arrow). This was correctly interpreted as metastasis.

tient). A total of 27 women and 33 men were evaluated; 27 patients were treated for primary colon cancer or local recurrence of a previously treated colorectal carcinoma, and 33 patients underwent operation for isolated liver metastases, 32 for elective resection and one for se- lective hepatic artery infusion. All patients underwent laparotomy. Before undergoing laparotomy, all patients underwent preoperative evaluation with 3.5 and $5 \mathrm{MH}$ ultrasonography (128 XP; Acuson, Mountain View, Calif.), conventional and contrast medium-enhanced 
CT (Somatom Plus; Siemens, Erlangen, Germany) with $5 \mathrm{~mm}$ contiguous sections, 1 -second scanning time, and interscanning delay of 5 seconds. Contrast-enhanced CT with administration of nonionic jopromide (U1travist 300; Schering, Berlin, Germany) was performed in two phases: phase $1,50 \mathrm{ml}$ in 25 seconds; and phase $2,50 \mathrm{ml}$ in 100 seconds. Angiography and continuous CT angiography (CCTA) were performed with CT data samples for 24 seconds at the same section level after a 3-second injection of 10 to $20 \mathrm{ml}$ of contrast medium was initiated in the common hepatic artery as previously described. ${ }^{14}$ After the angiographic catheter was placed, the average total CCTA examination time was $50 \mathrm{~min}$ utes (range, 37 to 54 minutes).

Imaging modalities were used independently by different investigators, and description of data was performed without knowledge of other examination results of anamnestic data other than a history of colorectal carcinoma. The laparotomy was performed without knowledge of CCTA findings.

Intraoperative ultrasonography by means of a $5 \mathrm{MHz}$ transducer for small areas (610; Aloka, Tokyo, Japan) was performed by radiologists who had no knowledge of previous imaging data. For the liver surface the sonographic inspection was performed with the addition of a water-filled balloon. The intraoperative findings were correlated with findings at surgical palpation and visual inspection of surface lesions. For the location of the lesions the segmentation of the liver as described by Couinaud ${ }^{15}$ was used.

\section{RESULTS}

The standard of reference was the findings at laparotomy: palpation and visual inspection of the liver surface, and IOUS.

One hundred five liver metastases were identified at laparotomy in 37 patients; 23 patients had no metastases. Without IOUS 89 lesions were identified by palpation and visual inspection. Sixteen (15\%) lesions were detected with IOUS only. Four small superficial lesions on the liver surface were barely detectable by IOUS, even with the help of a water-filled balloon. In 16 patients (43 lesions) IOUS data were correlated with resected specimens ( $5 \mathrm{~mm}$ slices), and concordance was $100 \%$ that the lesions were metastatic. Most of the lesions were so superficially located that the macroscopic appearance and one or two positive biopsy specimen results were regarded as conclusive for metastasis. The 16 lesions detected only with IOUS were either metastases proven by biopsy or were of the same appearance as other metastases proven by biopsy. Most of the nonpalpable lesions were located in segments 7 or 8. Overall, 42 metastases were less than $1 \mathrm{~cm}$ in diameter. Sixty-five lesions were exclusively situated in the right liver lobe (segments V through VIII), 38 lesions

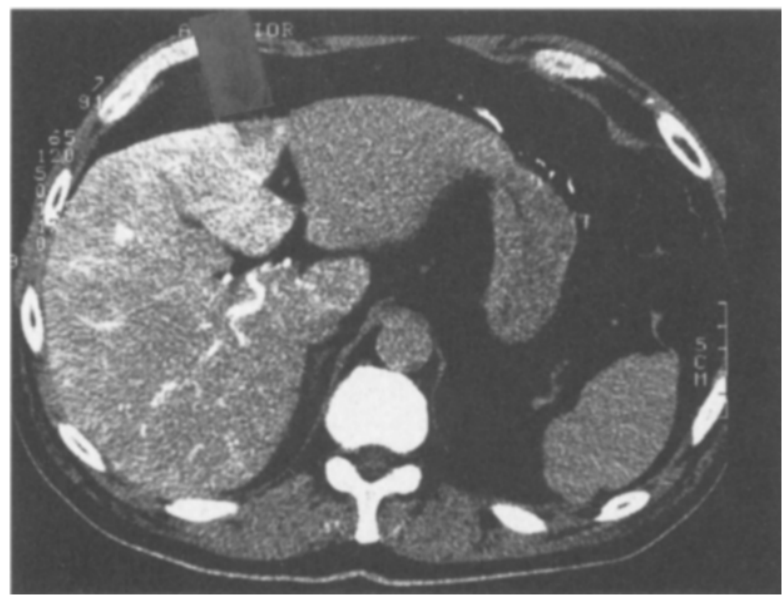

Fig. 2. Example of false-positive CCTA finding. Hypovascular solid lesion in segment 4 (arrow) was not metastasis but focal nodular hyperplasia.

were exclusively located in the left liver lobe (segments II through IV), and 2 lesions were found in the caudate lobe (segment I). Some lesions exclusively situated in segments V or VIII were also partially located in segment IV $\left(S_{4}\right)$.

CCTA enabled identification of $94 \%$ (99 of 105) of all lesions and was the most sensitive diagnostic modality $(p<0.001$ versus CT or ultrasonography) (Fig. 1). In four patients the left liver lobe $\left(S_{24}\right)$ or the left lateral segments $\left(\mathrm{S}_{2-3}\right)$ were not perfused at CCTA, and in one patient these unperfused segments contained two small metastases. The other four false-negative lesions at CCTA were all less than $1 \mathrm{~cm}$ in diameter and were superficially located in the left liver lobe $\left(S_{2-4}\right)$. With CCTA $90 \%$ (36 of 40) of the lesions with a diameter of $1 \mathrm{~cm}$ or less were detected. With CCTA all lesions $(n=62)$ in 11 patients with three or more metastases were correctly identified, whereas in only one patient with eight metastases two small metastases were missed. The percentages were far better than with ultrasonography and CT (Table I). Therefore in six patients a needless laparotomy could have been prevented if CCTA had been the standard of reference when you refrain from surgery when there are four or more metastases in case of planned hepatic resection or no metastases at all in case of planned resection for local recurrence.

CCTA, however, resulted in many false-positive findings (Table II, Fig. 2). Overall, 35 false-positive diagnoses were given with CCTA. Pathologic examination of liver resection specimens with 12 false-positive lesions revealed that in five cases a protrusion of the main metastasis was incorrectly interpreted as a separate metastasis, whereas in one patient focal nodular hyperplasia was found. Normal liver parenchyma was found in six other false-positive cases. The other false-positive lesions could not be verified with transection; they could be 
Table I. Sensitivity of preoperative diagnostic techniques according to size and number of lesions

\begin{tabular}{lcc} 
Techniques & $\begin{array}{c}\text { All lesions } \\
(n=105)(\%)\end{array}$ & $\begin{array}{c}\text { Lesions }<1 \mathrm{~cm} \text { in } \\
\text { diameter }(n=42)(\%)\end{array}$ \\
\hline Ultrasonography & $50(48)$ & $6(14)$ \\
CT & $55(52)$ & $3(7)$ \\
CCTA & $99(94)^{*}$ & $36(86)^{*}$ \\
\hline
\end{tabular}

*CCTA was significantly $(p<0.001)$ better than CT and ultrasonography in detection of metastases (chi-squared analysis)

verified with IOUS and surgical inspection and palpation only. In one patient two cysts had been incorrectly interpreted as metastases in a liver with nine real metastases. In two patients (two false-positive lesions) a recent scar in the liver tissue (excision biopsy site a few months before) was probably the cause of the misjudgment. One false-positive lesion was probably also a nonexisting satellite lesion of a large metastasis; the other lesions were probably all perfusion abnormalities in sometimes very inhomogeneously attenuating liver parenchyma. The false-positive lesions with CCTA look in most cases like round, nonenhancing lesions. A small minority were round, enhancing, and sometimes ringlike. $\mathrm{Al}$ most all lesions were less than $15 \mathrm{~mm}$ in diameter. This finding means that with CCTA 10 patients would not have undergone a needless operation. Specifications of these 10 patients are outlined in Table III.

Sensitivity, specificity, accuracy, and positive and negative predictive values are given in Table IV. Overall, CCTA had the highest accuracy of all methods $(74 \%$, or 118 of 159 diagnoses) (Table IV).

\section{DISCUSSION}

Hepatic resection has obtained a definitive place in the treatment of (isolated) liver metastases from colorectal cancer. ${ }^{1-3}$ The most important parameters predicting success for hepatic resection are whether the number of metastatic deposits do not exceed three or four and whether tumor-free margins can be obtained. ${ }^{1-3}$ Therefore imaging methods must be capable of providing the information necessary to choose among potentially successful interventions. The most important task of preoperative imaging before a planned laparotomy is to prevent a needless operation. During the past decade the standard modalities for preoperative evaluation of hepatic metastases have been CT and ultrasonography. ${ }^{4,5}$ However, comparison of CT and ultrasonography data with surgical findings and IOUS has shown a frustratingly low sensitivity of CT and ultrasonography for correct diagnosis of all metastatic lesions. $^{6-8}$ Combined IOUS and palpation are the most accurate methods in the detection of hepatic metastases. ${ }^{8}{ }^{16}$ IOUS has limitations in the identification of
Table II. Distribution of negative and positive diagnosis of metastasis $(n=105)$ by imaging techniques with standard of reference:

IOUS + surgical palpation

\begin{tabular}{lcccc}
\hline Technique & $\begin{array}{c}\text { True } \\
\text { negative }\end{array}$ & $\begin{array}{c}\text { False } \\
\text { negative }\end{array}$ & $\begin{array}{c}\text { True } \\
\text { positive }\end{array}$ & $\begin{array}{c}\text { False } \\
\text { positive }\end{array}$ \\
\hline Ultrasonography & 23 & 55 & 50 & 1 \\
CT & 20 & 50 & 55 & 7 \\
CCTA & 19 & 6 & 99 & 35 \\
\hline
\end{tabular}

small surface lesions, as we experienced four times. Conversely, palpation is limited in the detection of small subsurface lesions. In this study a lesion was considered positive only if interpreted as a metastasis by means of IOUS examination by a radiologist of a positive identification by surgical palpation. In 26 patients ( 43 lesions) intraoperative findings were correlated with resected specimens, and there was a $100 \%$ concordance that the lesions were metastatic.

For the other lesions, ideally, biopsy would be used to inform or refute malignancy for every lesion. We chose an approach to perform biopsies on all questionable lesions and those that were dissimilar to a biopsy-proven or obviously malignant lesion. This approach formed the standard used to assess the individual diagnostic modalities. The preoperative modalities were used independently by different investigators, with no other anamnestic knowledge than a history of colorectal carcinoma. In addition, the surgeons and the radiologists who performed IOUS were not informed of the results of CCTA.

In this study application of ultrasonography and CT led to sensitivity figures of only $48 \%$ for ultrasonography and 52\% for CT. Most of the missed lesions are smaller than $1 \mathrm{~cm}$ in diameter. The sensitivities of ultrasonography and CT make them unsuitable for accurate preoperative imaging. Therefore to detect small lesions and examine the liver thoroughly when resection of hepatic metastases is being considered, other investigative procedures are needed. In this study a new diagnostic technique based on CT arteriography was used. ${ }^{14}$

Selective arterial contrast-enhanced CT has been reported to provide a much higher lesion detection sensitivity, $9,13,17$ and in this study we used a modification of this technique.

A very high sensitivity (overall $94 \%$ ) in detection of liver metastases was obtained, and even $90 \%$ of the lesions with a diameter of $1 \mathrm{~cm}$ or less were detected. Conversely, the false-positive rate of this technique was very high.

Most of the false-positive interpretations were probably caused by perfusion abnormalities. Most lesions were round and nonenhancing, not wedge-shaped or geo- 
Table II. False-positive CCTA data in 10 patients who would not have undergone useful operation

\begin{tabular}{|c|c|c|c|c|c|c|c|c|}
\hline \multirow[b]{2}{*}{ Patient no. } & \multirow[b]{2}{*}{$M / F$} & \multirow[b]{2}{*}{ Disease } & \multicolumn{4}{|c|}{ No. of metastases found } & \multirow[b]{2}{*}{ Resection } & \multirow[b]{2}{*}{ CCTA problems } \\
\hline & & & Ultrasonography & $C T$ & CCTA & Real & & \\
\hline 1 & $\mathrm{~F}$ & LR & - & - & 2 & - & + & Perfusion \\
\hline 2 & M & $\mathrm{HM}$ & 2 & 2 & 4 & 2 & HHR & Protrusions* \\
\hline 3 & M & LR & $一$ & - & 2 & - & + & Perfusion \\
\hline 4 & M & HM & 2 & 2 & 4 & 2 & HHR & $1 \times$ protrusion $*$ \\
\hline 5 & M & HM & 1 & 1 & 5 & 3 & $\mathrm{~S}_{2+3}$, Wedge $\mathrm{R}$ & Perfusion \\
\hline 6 & M & HM & - & 1 & 6 & 1 & $\mathrm{~S}_{6}$ & Perfusion \\
\hline 7 & $\mathbf{F}$ & LR & - & - & 1 & 一 & + & Perfusion \\
\hline 8 & M & HM & 2. & 3 & 6 & 3 & HHR & Perfusion* \\
\hline 9 & M & HM & 2 & 2 & 5 & 2 & HHR & $1 \times$ protrusion $*$ \\
\hline 10 & M & HM & 1 & 1 & 4 & 1 & $\mathrm{HHL}$ & Perfusion \\
\hline
\end{tabular}

$F$, Female; $M$, male; $L R$, local recurrence; $H M$, hepatic metastasis; $H H R / H H L$, hemihepatectomy right/left.

*False-positive lesions in resected sliced specimen.

Table IV. Probability data for identification of metastases in percentages

\begin{tabular}{lccccc}
\hline Technique & Sensitivity & Specificity & Accuracy & $\begin{array}{c}\text { Positive } \\
\text { predictive } \\
\text { value }\end{array}$ & $\begin{array}{c}\text { Negative } \\
\text { predictive } \\
\text { value }\end{array}$ \\
\hline Ultrasonography & 48 & 96 & 57 & 98 & 29 \\
CT & 52 & 74 & 57 & 89 & 29 \\
CCTA & 94 & 35 & 74 & 74 & 76 \\
\hline
\end{tabular}

graphic $^{13}$ (Fig. 2). The assumption that experience with image interpretation will lead to a more accurate description with less false-positive lesions was not valid, because an interim analysis of 30 patients did not lead to a better specificity. Therefore arteriographically enhanced CT leads to certain types of perfusion abnormalities that are confused with tumor neovascularization. Some authors have recommended CT during arterial portography (CTAP). ${ }^{10,11,18}$ The metastasis detection rate for CTAP is usually better than that of CTA, although not as good as in our study with CCTA. The specificity of CTAP was better in some studies, ${ }^{10,11,19}$ although this finding is not true in all studies. ${ }^{20}$ In general, CTAP is also marred by a high false-positive rate resulting from laminar flow perfusion defects, although possibly less than with CCTA. ${ }^{13}$ However, an advantage of CTAP is a more accurate localization of metastases into hepatic segments. ${ }^{13}$ Magnetic resonance imaging is also a new imaging technique that is more sensitive than conventional CT scanning, but it does not seem to be as sensitive as CCTA of CTAP. ${ }^{12,21}$ In the Far East, CT scanning performed after lipiodol (an iodinated oil) is injected via a catheter placed in the hepatic artery can also show small liver lesions. The technique, however, is mainly used in case of primary liver cancer nodules. ${ }^{13}$

For decision making it seems necessary that CCTA data are verified. It may be true that better accuracies can be obtained by combining preoperative data. How- ever, the general inability of ultrasonography and conventional CT to detect small lesions (less than $1 \mathrm{~cm}$ ) hampers success by combination of data. What cannot be seen cannot be combined. Combining CCTA data with intraoperative sonography seems to be the most useful method. In this study the IOUS examination was performed by an investigator who did not know the CCTA data. IOUS could confirm correct positive CCTA data, so the needless laparotomy in six patients would have been prevented. Of more importance is that to eliminate false-positive CCTA findings, IOUS is also highly necessary. A purely diagnostic IOUS, perhaps performed by a laparoscopic approach, might be useful to try. However, in quite a number of patients adequate IOUS data can be obtained only after the liver has been fully mobilized, and for that a formal laparotomy is necessary.

In conclusion, CCTA is an accurate technique to detect liver metastases, but in our opinion it is not good enough to merit routine use. It is also a very time-consuming procedure (average examination time $50 \mathrm{~min}$ utes after angiographic catheter placement). CCTA cannot be used as a routine technique for determining the feasibility of hepatic resection. CTAP could alter this statement if specificity is markedly higher then CCTA. This possibility is currently under investigation. In a recent review on liver tumor imaging, Ferruci ${ }^{13}$ stated that at present the preferred method is CTAP. It would appear that the major role for CCTA is to prevent some 
needless laparotomies in patients at high risk such as those with local recurrences in whom a negative result of a CCTA scan might help with the decision to proceed with operation.

\section{REFERENCES}

1. Adson MA. Resection of liver metastases: when is it worthwhile? World J Surg 1987;11:511-20.

2. Steele G, Ravikumar TS. Resection of hepatic metastases from colorectal cancer. Ann Surg 1989;210:127-38.

3. Van Ooijen B, Wiggers $T$, Meijer S, et al. Hepatic resections for colorectal metastases in the Netherlands. Cancer 1992;70:2834.

4. Smith TJ, Kemeny MM, Sugarbaker $\mathrm{PH}$, et al. A prospective study of hepatic imaging in the detection of metastatic disease. Ann Surg 1982;195:486-91.

5. Schreve RH, Terpstra OT, Ausema L, Lameris JS, van Seijen AJ, Jeekel J. Detection of liver metastases: a prospective study comparing liver enzymes, scintigraphy, ultra-sonography and computed tomography. Br J Surg 1984;71:947-9.

6. Clarke MP, Kane RA, Steele G, et al. Prospective comparison of preoperative imaging and intraoperative ultrasonography in the detection of liver tumors. Br J Surg 1989;76:1323-9.

7. Machi J, Isomoto N, Kurohiji T, et al. Accuracy of intra-operative ultrasound in diagnosing liver metastases from colorectal cancer: evaluation with postoperative follow-up results. World J Surg 1991;15:551-7.

8. Knol JA, Marn CS, Francis IR, Rubin JM, Bromberg J, Chang AE. Comparisons of dynamic infusion and delayed computed tomography, intraoperative ultrasound, and palpation in the diagnosis of liver metastases. Am J Surg 1993; 165:81-8.

9. Sitzmann JV, Coleman J, Pitt HA, et al. Preoperative assessment of malignant hepatic tumors. Am J Surg 1990;159:137-43.
10. Peterson MS, Baron RL, Dodd GD, et al. Hepatic parenchymal perfusion defects detected with CTAP: imaging-pathologic correlation. Radiology 1992; 185:149-55.

11. Soyer P, Levesque M, Elias D, Zeitoun G, Roche A. Detection of liver metastases from colorectal cancer: comparison of intraoperative US and CT during arterial portography. Radiology 1992;183:541-4.

12. Soyer P, Levesque M, Caudron C, Elias D, Zeitoun G, Roche A MRI of liver metastases from colorectal cancer vs CT during arterial portography. J Comput Assist Tomogr 1993;17:67-74.

13. Ferruci JT. Liver tumor imaging. Current concepts. Radiol Clin North Am 1994;32:39-54

14. Oudkerk M, van Ooijen B, Mali SPM, Tjiam SL, Schmitz PIM, Wiggers T. Liver metastases from colorectal carcinoma: detection with continuous CT angiography. Radiology 1992;185:157-61.

15. Couinaud C. Le foie; etudes anatomiques et chirurgicales. Paris: Masson, 1957.

16. Charnley RM, Morris DL, Dennison AR, Amar SS, Hardcastle JD. Detection of colorectal liver metastases using intraoperative ultrasonography. Br J Surg 1991;78:45-8.

17. Balfe DM. Hepatic metastases from colorectal cancer: radiologic strategies for improved selection. Radiology 1992;185:18-9.

18. Small WC, Mehard WB, Langmo LS, et al. Preoperative determination of the resectability of hepatic tumors: efficacy of CT during arterial portography. AJR 1993;161:319-22.

19. Nelson RC, Chezmar JL, Sugarbaker PH, Murray DR, Bernardino ME. Preoperative localization of focal liver lesions to specific liver segments: utility of CT during arterial portography. Radiology 1990;176:89-94

20. Miller DL, Simmons JT, Chang R, et al. Hepatic metastasis detection: comparison of three CT contrast enhancement methods. Radiology 1987;165:785-90.

21. Collier BD, Foley WD. Current imaging strategies for colorectal cancer. J Nucl Med 1993;34:537-40. 